\title{
Biologic therapy and tuberculosis: our experience and review of literature
}

\author{
Caterina Fabroni' \\ Angelo Massimiliano \\ D'Erme ${ }^{2}$ \\ Torello Lotti ${ }^{3}$ \\ 'Department of Dermatology, \\ Misericordia e Dolce Hospital, Prato, \\ Italy; ${ }^{2}$ Division of Dermatology, \\ Department of Surgery and \\ Translational Medicine, University \\ of Florence, Florence, Italy; ${ }^{3}$ Guglielmo \\ Marconi University, Rome, Italy
}

This article was published in the following Dove Press journal:

Psoriasis: Targets and Therapy

2 July 2013

Number of times this article has been viewed

\begin{abstract}
Psoriasis is an inflammatory dermatologic disease that can often involve not only the skin and mucosal surfaces but also the bones and joints. It affects approximately $3 \%$ of the world's population, and has a chronic-relapsing course. The management of psoriasis is often difficult and, particularly in the case of moderate to severe forms, the use of systemic therapies is mandatory. The introduction of biologic drugs has greatly improved our ability to treat psoriasis. However, it is necessary to underline that antitumor necrosis factor- $\alpha$ treatments may reactivate latent tuberculosis infection and cause particularly disseminated or extrapulmonary disease. Physicians should carry out proper screening of patients before starting treatment with antitumor necrosis factor- $\alpha$, in order to identify individuals with latent tuberculosis infection. We report our experience in this field and review the various existing tests to screen for tuberculosis.
\end{abstract}

Keywords: infliximab, adalimumab, tuberculosis, psoriasis

\section{Introduction}

Psoriasis is a hyperproliferative cutaneous disorder in which both adaptive and innate immunity plays an important role. Traditionally, the treatment of moderate to severe psoriasis has been limited by the safety of many of the drugs that are effective in clearing the disease. As greater understanding of the immunopathology of psoriasis has developed, more targeted therapies have emerged with apparently fewer toxic side effects. The biologic agents act on specific steps in the immunologic cascade to control psoriasis, and now account for a significant proportion of systemic therapies commonly used for psoriasis. ${ }^{1}$ In particular, psoriasis improves with the use of tumor necrosis factor- $\alpha$ (TNF $\alpha$ ) blockers, because TNF $\alpha$ plays a critical role in the pathogenesis of psoriasis, being increased in both skin lesions and sera of patients. TNF $\alpha$ mediates keratinocyte proliferation, cell adhesion, and trafficking to lesional sites, and stimulates growth and invasiveness of dermal fibroblasts. ${ }^{2-5}$

The TNF $\alpha$ antagonist infliximab is a murine-human chimeric monoclonal antibody constructed from murine and human DNA sequences comprising a mouse variable region and a human immunoglobulin G1- $\alpha$ constant region. Infliximab neutralizes the biologic activity of TNF $\alpha$ by binding with high affinity to the soluble and membranebound TNF $\alpha$ forms, thereby inhibiting binding of TNF $\alpha$ with its receptors, ${ }^{4}$ and has been shown to be highly effective for the treatment of psoriasis, producing rapid and dramatic decreases in epidermal inflammation and normalization of keratinocyte differentiation in psoriatic plaques that precede maximal clinical response. ${ }^{5}$

Treatment with biologics, especially those with anti-TNF $\alpha$ factors, is absolutely contraindicated in patients with active, serious infections. All of the TNF $\alpha$
Correspondence: Caterina Fabroni

UO Dermatologia, Ospedale Misericordia e Dolce, Prato, Italy

Tel +390574 434030

Fax +390574 434030

Email fabroni@dada.it 
inhibitors carry the potential for an increase of serious infections, particularly those that have been associated with an increased incidence in granulomatous infections, because TNF $\alpha$ is necessary for the development of the granulomatous response playing an important role in host defense against intracellular pathogens such as Mycobacterium tuberculosis, Listeria monocytogenes, and Histoplasma capsulatum. ${ }^{6-13}$ Overall, the risk of granulomatous infections in infliximab or adalimumabtreated patients is greater than in etanercept-treated patients. The ability in vivo to induce apoptosis of monocytes and $\mathrm{T}$ cells that constitute granuloma may, in part, explain the disparity of why infliximab is effective in the treatment of chronic granulomatous conditions such as Crohn's disease, but yet is associated with a higher incidence of infections from pre-existing granuloma. ${ }^{14}$

One-third of the world's population is infected with tuberculosis; nearly $1 \%$ is infected each year. New tuberculosis and other mycobacterioses outbreaks have occurred in Eastern Europe, where tuberculosis is increasing after almost 40 years of steady decline. Globally, factors other than the HIV/AIDS epidemic that have contributed to the rise in tuberculosis include poorly managed tuberculosis control programs, the increased movement of refugees and displaced people from developing countries, but also the wide spreading of immunosuppressive drugs in transplanted patients or those who are treated for immunologic diseases. ${ }^{15}$ Patients who are given anti-TNF $\alpha$ therapies may be at risk for both acquisition of primary tuberculosis and the reactivation of latent tuberculosis. Pharmacovigilance studies have also confirmed that anti-TNF $\alpha$ increases the risk of tuberculosis, and that infliximab and adalimumab carry a higher risk than etanercept. ${ }^{16-18}$

The immunosuppression due to anti-TNF $\alpha$ in such patients not only masks the symptoms and signs of tuberculosis but also predisposes the patients to more severe variants of the disease. In fact, more than half of cases $(52 \%)$ onset during infliximab treatment had extrapulmonary tuberculosis, and 24\% had disseminated tuberculosis. ${ }^{18,19}$ While being treated with biologics, patients need to be periodically evaluated for the development of new symptoms and/or signs of tuberculosis infection or reactivation, and if patients show these symptoms while being treated with a biologic agent, it is prudent to withhold the biologic and to begin antitubercular therapy.

Until now, internationally approved guidelines have included only the tuberculin skin test (TST) (Mantoux reaction) and chest radiograph for tuberculosis screening, but this screen often offers false-negative results. For this reason, in recent years new experimental assays have been tested. ${ }^{15,18-21}$ The chest radiograph is a good screening tool for active tuberculosis. Radiologic findings of active pulmonary tuberculosis include lung infiltrates, patchy bronchopneumonia, cavitation, and a miliary pattern. The chest radiograph may show abnormalities suggestive of prior tuberculosis. Nodules and scars may contain slowly multiplying tubercle bacilli with substantial potential for future progression to active disease.

TST is a measure of the individual's delayed hypersensitivity response to the tuberculosis bacilli, and until 2001 was the only test used to diagnose latent tuberculosis infection (LTBI). This assay had its disadvantages: low sensitivity with a risk of a false negative, especially in immunosuppressed subjects and those in the early phase of infection (it is worth noting that in an immunocompetent person, 4-12 weeks are needed after infection to have an immune response to tuberculin). This test is also operator-dependent and requires two visits within 48-72 hours for assay interpretation and boosting phenomena. For all these reasons, tuberculosis surveillance programs have sought a better diagnostic tool, the interferon- $\gamma\left(\right.$ IFN- $\gamma$ ) blood test QuantiFERON ${ }^{\circledR}$-TB (QFT) (Cellestis Limited, Carnegie, Victoria, Australia), approved by the US Food and Drug Administration in 2001, as an aid for detecting LTBI. This assay is an in vitro diagnostic aid and is based on the quantification of IFN- $\gamma$ released by sensitized lymphocytes in whole blood incubated for 16-24 hours with purified protein derivative (PPD), an antigen of $M$. tuberculosis. After incubation, the concentration of IFN- $\gamma$ in the separated plasma is determined by enzyme-linked immunosorbent assay.

Nowadays there is a new IFN- $\gamma$ test, the QuantiFERON ${ }^{\circledR}$ TB Gold (QFTG), licensed in 2005. The QFTG measures the IFN- $\gamma$ release in response to synthetic peptides, which simulate the two peptides present in PPD. It differs from QFT in that it measures the IFN- $\gamma$ release in response to PPD. The QFTG was approved as an aid in detecting LTBI. The advantages of QFTG compared with the TST are that there is a very low risk of booster phenomenon, a similar specificity and sensitivity as Mantoux reaction, and no operator dependence. ${ }^{22}$ On the other hand, QFTG is more expensive than TST; cannot be used in some patient groups, such as children aged younger than 17 years and pregnant women; requires access to a laboratory performing QFTG; and cannot differentiate infection with tuberculosis disease from LTBI. ${ }^{21-27}$ Lastly, the "wobble" phenomenon, in which 
repeat testing reveals conversions and reversions around the cutoff, has to be remembered.

\section{Case report}

Between 2006 and 2011 we have treated more than 600 patients (about 120 new patients/year) with anti-TNF $\alpha$ drugs (etanercept, infliximab, adalimumab). Before starting the treatment, all patients were screened using TST and a chest radiograph to detect tuberculosis infection. A chemistry screen also included liver function tests; complete blood cell count, including platelet count; and hepatitis B and C markers.

In our experience, we observed only three cases of development of severe opportunistic infections during therapy with an anti-TNF $\alpha$. We reported a case of sporotrichosis during treatment with etanercept, and two cases, respectively, of tuberculosis and legionellosis during treatment with infliximab.

Particularly, we report the case of a 37-year-old man, a patient affected by a severe form of psoriasis refractory to the traditional drugs (oral retinoids, psoralen and ultraviolet $\mathrm{A}$ therapy, and cyclosporin) (Figure 1). Before starting therapy with infliximab, blood tests and radiologic findings were normal and TST was negative. After 4 months of treatment, we observed a dramatic improvement in skin lesions (Figure 2),
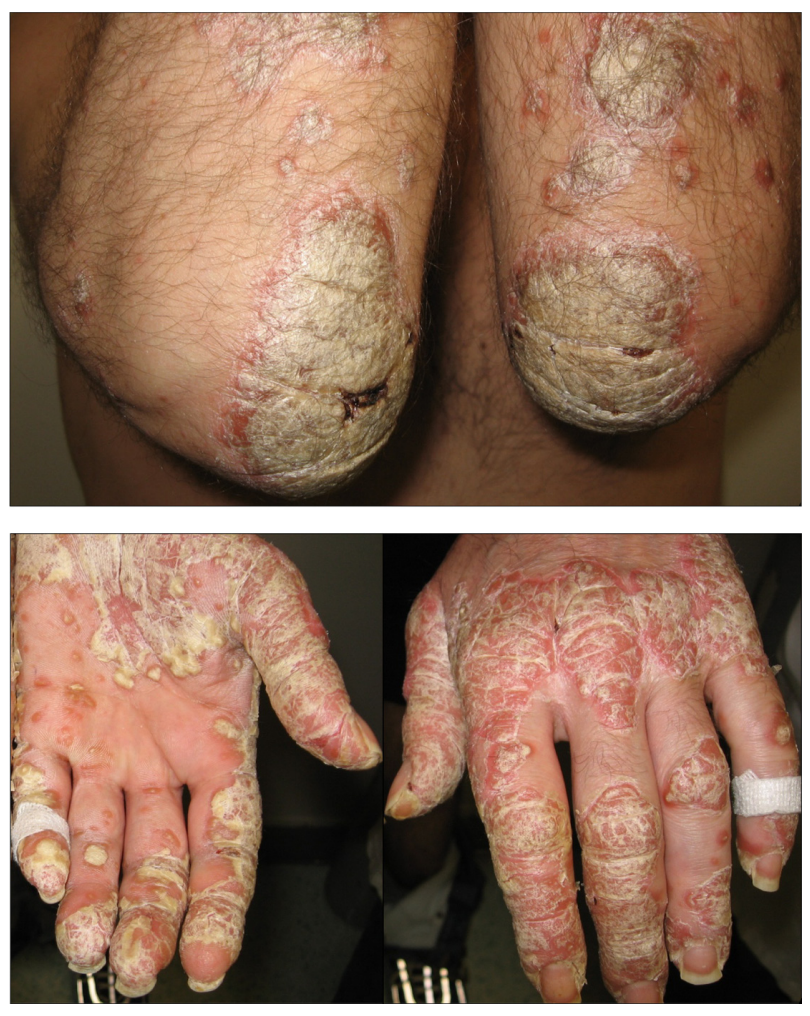

Figure I Skin lesions before treatment.
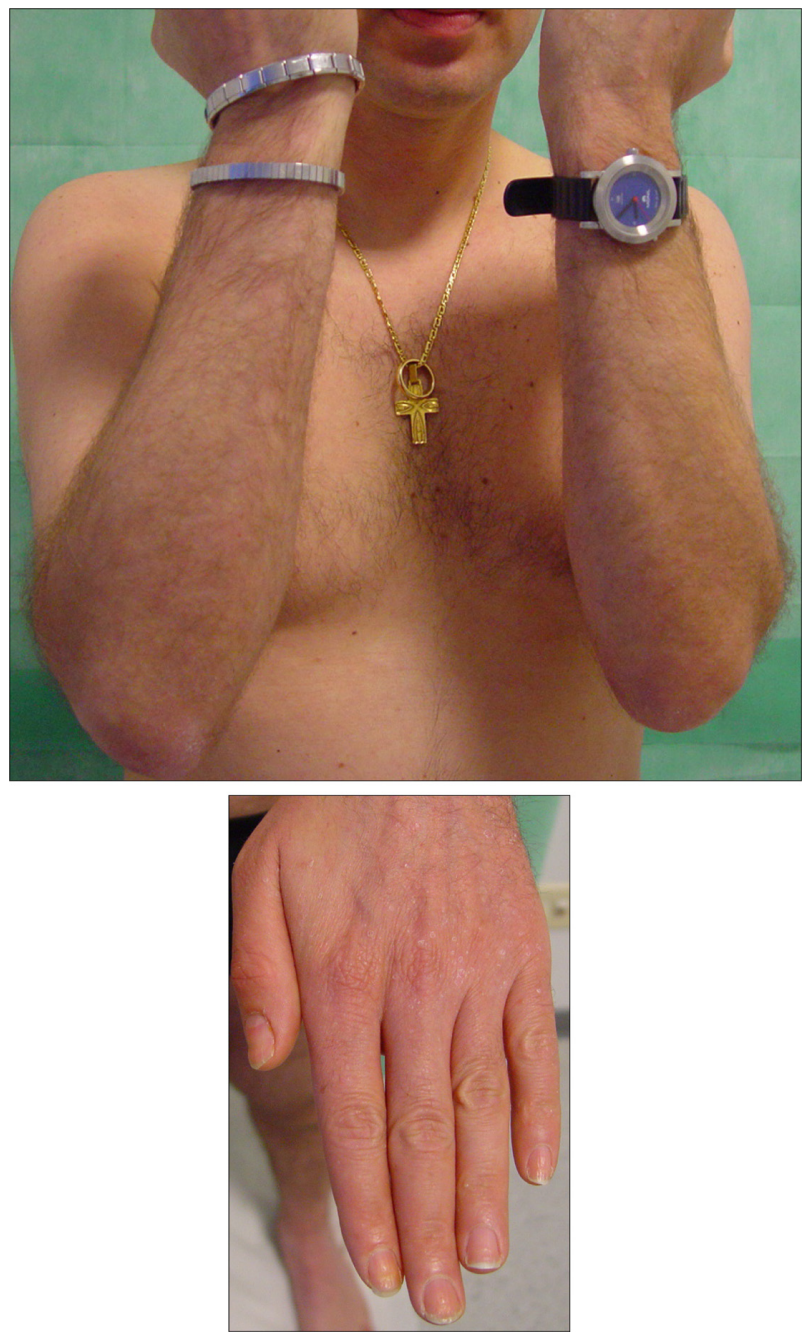

Figure 2 Skin lesions after 3 months of treatment with infliximab.

but the patient presented persistent and antibiotic-resistant fever with night sweats and severe headache with lipothymia. He was admitted to the Infectious Disease Department. During hospitalization, a chest radiograph showed a globular thickening at the left pulmonary hilum. Antibiotic therapy with ceftriaxone and azithromycin was administered with little improvement. TST, $M$. tuberculosis culture from sputum, and QFTG results were negative. Computed tomography (CT) of the chest revealed a diffuse micronodular pattern of the antero-lateral segment of the left lower pulmonary lobe and multiple mediastinic lymphadenopathies. In addition, the CT scans presented a pleural effusion in the right lower lung lobe connected with lamellar and calcified pulmonary atelectasia and a pericardial effusion. A CT scan of the abdomen showed diffuse mesenteric adenopathies. The histopathologic examination of the mediastinic lymphonode showed a necrotic and colliquative flogosis with caseation degeneration and gigantocellular granulomas. The previous 
suspicion of tuberculosis infection was finally confirmed by histopathology.

The patient underwent antitubercular therapy (isoniazid, rifampicin, ethambutol, and pyrazinamide), and in a few days all symptoms progressively improved. Microscopic examination and polymerase chain reaction on sputum smear became positive after 45 days, whereas TST and QFTG results were again negative.

\section{Conclusion}

Anti-TNF $\alpha$ therapies have a high tendency to stimulate granulomatous infections, and therefore patients with associated comorbidities have to be very careful. Among the first marketed anti-TNF $\alpha$ antagonists, the higher risk of tuberculosis has been reported for infliximab and adalimumab (than for etanercept). TNF $\alpha$ stimulates the production of cytokines as such as interleukin-2, -3, -4, and -8 in macrophages and T cells. All these soluble factors enhance keratinocyte hyperproliferation and the expression of CD54, and increase the phagocytic capacity of macrophages. Moreover, infliximab induces granulomatous cell apoptosis linking the membrane-bound TNF $\alpha$. As well as an immunologic action, TNF $\alpha$ is able to enhance the killing of intracellularly viable bacteria through the production of reactive nitrogen and oxygen species, synergizing with IFN- $\gamma$, and also may be involved in the maintenance of macrophage viability at the site of infection. ${ }^{1-3}$ Therefore, new infections should be closely monitored, and sometimes the therapy has to be stopped. ${ }^{28,29}$ Moreover, patients should be instructed in how to recognize signs and symptoms of infection and be advised to seek medical attention when they occur. Unfortunately, in our case, a close clinical and laboratory control program did not permit recognition of a tuberculosis reactivation or a new tuberculosis infection, because Mantoux reaction results were negative before starting infliximab therapy, and both TST and GFTG were negative during the active disease phase. In our case, only the mediastinic lymphonodal biopsy revealed tuberculosis infection. The reason for the unchanged QFTG results in our patient, also during the active disease phase, is not understood. Our hypothesis is that the reduction of soluble IFN $\gamma$, which is downregulated during anti-TNF therapy, could allow the widespread infection of $M$. tuberculosis without a positive QFTG and Mantoux test. A second hypothesis is that the standard quantity of test antigens would not be enough to stimulate a significant immune response. Finally, the patient could have contracted tuberculosis late during therapy, and the tests would be false-negative because the infection takes from 4 to 12 weeks to produce a delayed hypersensitivity response.
Despite QTFG and TST tests showing good sensitivity, ${ }^{29,30}$ we want to highlight an example of evidence of the unreliability of immunity tests, including the QTFG, in patients treated with anti-TNF $\alpha$ drugs affected by active tuberculosis.

This case underlines that physicians should be aware of the increased risk of reactivation of tuberculosis among people treated with anti-TNF $\alpha$ drugs, and should screen their patients to determine whether they have LTBI. Because tubercular screening tests may have false-negative results, a detailed assessment of the risk of tuberculosis should be performed in every case, also because immunodepression due to these drugs may increase the possibility of developing a miliary tuberculosis with exitus.

\section{Disclosure}

The authors report no conflicts of interest in this work.

\section{References}

1. Kipnis CD, Myers WA, Opeola M, Gottlieb AB. Biologic treatments for psoriasis. J Am Acad Dermatol. 2005;52:671-682.

2. Gaspari AA. Innate and adaptive immunity and the pathophysiology of psoriasis. J Am Acad Dermatol. 2006;54:S67-S80.

3. Krueger G, Callis K. Potential of tumor necrosis factor inhibitors in psoriasis and psoriatic arthritis. Arch Dermatol. 2004;140:218-225.

4. Laffitte E, Janssens JP, Roux-Lombard P, Thielen AM, Barde C, Marazza G, et al. Tuberculosis screening in patients with psoriasis before antitumour necrosis factor therapy: comparison of an interferon-c release assay vs tuberculin skin test. Br J Dermatol. 2009;161:797-800.

5. Gottlieb AB, Masud S, Ramamurthi R, Abdulghani A, Romano P, Chaudhari U, et al. Pharmacodynamic and pharmacokinetic response to anti-tumor necrosis factor- $\alpha$ monoclonal antibody (infliximab) treatment of moderate to severe psoriasis vulgaris. $J$ Am Acad Dermatol. 2003;48:68-75.

6. Wallis RS, Broder M, Wong J, Lee A, Hoq L. Reactivation of latent granulomatous infections by infliximab. Clin Infect Dis. 2005;41(Suppl): S194-S198.

7. Raychaudhuri S, Shmerling R, Ermann J, Helfgott S. Development of active tuberculosis following initiation of infliximab despite appropriate prophylaxis. Rheumatology (Oxford). 2007;46:887-888.

8. Sichletidis L, Settas L, Spyratos D, Chloros D, Patakas D. Tuberculosis in patients receiving anti-TNF agents despite chemoprophylaxis. Int $J$ Tuberc Lung Dis. 2006;10:1127-1132.

9. Winterfield LS, Menter A. Infliximab. Dermatol Ther. 2004;17: 409-426.

10. Ehlers S. Tumor necrosis factor and its blockade in granulomatous infections: differential modes of action of infliximab and etanercept? Clin Infect Dis. 2005;41:S199-S203.

11. Khanna D, McMahon M, Furst DE. Safety of tumour necrosis factor-a antagonists. Drug Saf. 2004;27(5):307-324.

12. Gardam MA, Keystone EC, Menzies R, Manners S, Skamene E, Long R. Anti-tumor necrosis factor agents and tuberculosis risk: mechanisms of action and clinical management. Lancet Infect Dis. 2003;3:148-155.

13. Arend SM, Breedveld FC, Van Dissel JT. TNF- $\alpha$ blockade and tuberculosis: better look before you leap. Neth J Med. 2003;61:111-119.

14. Wallis RS, Broder MS, Wong JY, Hanson ME, Beenhouwer DO. Granulomatous infectious diseases associated with tumor necrosis factor antagonists. Clin Infect Dis. 2004;38:1261-1265.

15. World Health Organization. Global tuberculosis control. WHO report 2001. Geneva: WHO; 2001. Document WHO/CDS/ tuberculosis/2001.287. 
16. Nacci F, Matucci-Cerinic M. Tuberculosis and other infections in the anti-tumour necrosis factor-alpha (anti-TNF- $\alpha$ ) era. Best Pract Res Clin Rheumatol. 2011;25(3):375-388.

17. Furst DE, Wallis R, Broder M, Beenhouwer DO. Tumor necrosis factor antagonists: different kinetics and/or mechanisms of action may explain differences in the risk for developing granulomatous infection. Semin Arthritis Rheum. 2006;36(3):159-167.

18. Metcalfe JZ, Everett CK, Steingart KR, et al. Interferon- $\gamma$ release assays for active pulmonary tuberculosis diagnosis in adults in low- and middle-income countries: systematic review and meta-analysis. J Infect Dis. 2011;204 Suppl 4: S1120-S1129.

19. Keane J, Gershon S, Wise RP, Mirabile-Levens E, Kasznica J, Schwieterman WD, et al. Tuberculosis associated with infliximab, a tumor necrosis factor-alpha neutralizing agent. $N \mathrm{Engl} \mathrm{J} \mathrm{Med.}$ 2001;345:1098-1104.

20. Keane J, Gershon SK, Braun MM. Tuberculosis and treatment with infliximab. N Engl J Med. 2002;346:623-626.

21. Tat D, Polenakovik H, Herchilne T. Comparing interferon- $\gamma$ release assay with tuberculin skin test readings at 48-72 hours and 144-168 hours with use of 2 commercial reagents. Clin Infect Dis. 2005;40(2):246-250.

22. Centers for Disease Control and Prevention. Guidelines for using the QuantiFERON-TB Gold test for detecting Mycobacterium tuberculosis infection, United States. MMWR. 2005;54(RR-15):49-55.

23. Mazurek GH, LoBue PA, Daley CL, Bernardo J, Lardizabal AA, Bishai WR, et al. Comparison of a whole-blood interferon gamma assay with tuberculin skin testing for detecting latent Mycobacterium tuberculosis infection. JAMA. 2001;286(14):1740-1747.
24. Pai M, Riley LW, Colford JM. Interferon-gamma assays in the immunodiagnosis of tuberculosis: a systematic review. Lancet Infect Dis. 2004;4:761-776.

25. Centers for Disease Control and Prevention. Guidelines for using QuantiFERON-TB test for diagnosing latent Mycobacterium tuberculosis infection. MMWR Recomm Rep. 2003;52(RR-2):15-18.

26. Chiu H-Y, Hsueh P-R, Tsai T-F. Clinical experience of QuantiFERON-TB Gold testing in patients with psoriasis treated with tumour necrosis factor blockers in Taiwan. Br J Dermatol. 2011;164:553-559.

27. Menzies D, Pai M, Comstock G. Meta-analysis: new tests for the diagnosis of latent tuberculosis infection: areas of uncertainty and recommendations for research. Ann Intern Med. 2007;146:340-354.

28. Fabroni C, Gori A, Prignano F, Lotti T. A severe complication of antiTNF alfa treatment. G Ital Dermatol Venereol. 2010;145(6):775-777.

29. Rutherford M, Alisjahbana B, Maharani W, Sampurno H, van Crevel R, Hill PC. Sensitivity of the QuantiFERON-Gold in-tube assay in sputum smear positive TB cases in Indonesia. PLoS One. 2010;5(8):e12020.

30. Gori A, Fabroni C, Prignano F, Lotti T. Unusual presentation of tuberculosis in an infliximab-treated patient - which is the correct TB screening before starting a biologic? Dermatol Ther. 2010;23 Suppl 1 $\mathrm{S} 1-\mathrm{S} 3$.
Psoriasis: Targets and Therapy

\section{Publish your work in this journal}

Psoriasis: Targets and Therapy is international, peer-reviewed, open access journal focusing on psoriasis, nail psoriasis, psoriatic arthritis and related conditions, identification of therapeutic targets and the optimal use of integrated treatment interventions to achieve improved outcomes and quality of life. The manuscript management system

\section{Dovepress}

is completely online and includes a very quick and fair peer-review system. Visit http://www.dovepress.com/testimonials.php to read real quotes from published authors. 